\title{
Poultry fungal contamination as a public health problem
}

\author{
C. Viegas ${ }^{1}$, C. Veríssimo ${ }^{2}$, L. Rosado ${ }^{2}$ \& C. Silva Santos ${ }^{3}$ \\ ${ }^{1}$ Higher School of Health Technologies of Lisbon, \\ Polytechnic Institute of Lisbon, Portugal \\ ${ }^{2}$ National Institute of Health Dr. Ricardo Jorge, \\ Mycology Laboratory, Portugal \\ ${ }^{3}$ School of Public Health, New University of Lisbon, Portugal
}

\begin{abstract}
A descriptive study was developed to monitor poultry fungal contamination. Five air samples of 100 litres through impaction method were collected and 4 swab samples from surfaces were also collected using a $10 \mathrm{~cm}$ square of metal. Simultaneously, temperature and humidity were monitored as well. Twenty different species of fungi in air were identified, being the 4 most commonly isolated the following genera: Cladosporium (40,5\%), Alternaria (10,8\%), Chrysosporium and Aspergillus (6,8\%). Concerning surfaces, 21 different species of fungi were identified, being the 4 genera more identified Penicillium (51,8\%), Cladosporium (25,4\%), Alternaria (6,1\%) and Aspergillus (4,2\%). In addition, Aspergillus flavus also isolated in the poultry air, is a well-known producer of potent mycotoxins (aflatoxins) and besides this species other isolated genera, like Fusarium and Penicillium, are also known as mycotoxins producers. Also noteworthy is the fact that Aspergillus fumigatus, one of the species isolated in air and surfaces, is one of the saprophytic fungi most widespread in air and is capable of causing severe or sometimes fatal aspergillosis. There was no significant relationship $(\mathrm{p}>0,05)$ between fungal contamination and temperature and humidity.
\end{abstract}

Keywords: poultry, fungal contamination, mycotoxins, public health problem.

\section{Introduction}

Fungi presence requires ideal conditions of temperature, humidity, oxygen, carbon sources, nitrogen and minerals. Their biological activities of 
biodegradation and biodeterioration, depend on their enzymes activity, the environmental conditions, the competition phenomenon and the nature of the substrate. In situations where the fungal concentrations are high or when people suffer from respiratory problems or have a weak immune system, exposure to fungi can cause the onset of symptoms and disease. The effects are dependent on the species present, the metabolic products, the concentration and exposure duration and individual susceptibility [1].

Until now, epidemiological studies have failed to establish a causal relationship of the extent of fungal presence, exposure time and specific effects on health or frequency and severity of symptoms reported. Studies tend to show only existence of a link between exposure to fungi and development of symptoms, especially respiratory symptoms [1]. However, fungal species are generally identified as the cause of allergic diseases, headaches, eye irritation, obstruction of airways, coughing and other symptoms [2].

In addition, a group of indoors molds can produce secondary metabolites, like mycotoxins, in response to changes in their environment. Mycotoxins can be pro-inflammatory, immunosuppressive or carcinogenic [3]. The different chemical groups of mycotoxins include aflatoxins, fumonisins, ochratoxins, rubratoxins, and trichothecene toxins, all with different biologic properties [3]. Agricultural operations, such as animal feeding, increase farmers' risk of exposures to airborne dust and micro organisms like fungi [4]. Besides that, in Portugal there is an increasingly industry of large facilities that produce whole chickens for domestic consumption. Although much research has been done on microbial contaminants associated with the various stages of processing poultry and meat products $[5,6]$, only few investigations have reported on the indoor air of these plants [7,8]. Moreover, air plays a significant role in the poultry meat contamination [7] and there is evidence that mycotoxins can cause human disease from the ingestion of fungus-contaminated food [9].

This investigation was designed to describe in one poultry environmental fungal contamination phenomena and explore possible associations with independent environmental variables.

\section{Materials and methods}

A descriptive study was developed to monitor one poultry fungal contamination. Five air samples of 100 litres each through impaction method were collected and 4 swab samples from surfaces were also collected using a $10 \mathrm{~cm}$ square of metal. Simultaneously, two environmental parameters - temperature and relative humidity - were monitored, using the Babouc equipment, (LSI Sistems), according to the International Standard ISO 7726 - 1998.

Air samples were collected at $140 \mathrm{~L} /$ minute and at one meter tall using malt extract agar with chloramphenicol as a bacteria growth inhibitor (MEA), in the facilities, and also, outside premises, since this is the place regarded as reference. Swabs were performed according to the International Standard ISO 18593 2004, using a $10 \mathrm{~cm}$ square of metal disinfected with $70 \%$ alcohol solution between samples. Swabs were inoculated in triplicate on MEA and in mycobiotic 
agar with cycloheximide (MA). Subsequently, these were incubated at $27^{\circ} \mathrm{C}$ (MEA for 5 to 7 days and MA for 15 to 20 days).

After laboratory processing and incubation of the collected samples, quantitative $\left(\mathrm{CFU} / \mathrm{m}^{3}\right.$ and $\left.\mathrm{CFU} / \mathrm{m}^{2}\right)$ and qualitative results were obtained, with identification of isolated fungal species. Whenever possible, filamentous fungi were identified to the species level, since adverse health effects vary according to fungal species $[10,11]$. Identification of filamentous fungi was carried out on material mounted in lactophenol blue and achieved through morphological characteristics listed in illustrated literature [11].

With the obtained data, tables with frequency distribution of isolated fungal species were made. Fungal concentration dependence in the two monitored environmental parameters - temperature and relative humidity - was also analyzed.

\section{Results}

Twenty different species of fungi in air were identified, being the 4 most commonly isolated the following genera: Cladosporium (40,5\%), Alternaria (10,8\%), Chrysosporium and Aspergillus (6,8\%). Among Aspergillus genus, were identified the species Aspergillus flavus, Aspergillus niger and Aspergillus fumigatus. In addition to these genera, were also identified: Fusarium sp., Fusarium incarnatum, Fusarium oxysporum, Exophiala werneckii, Stemphylium sp., Exophiala sp., Phoma sp., Scytalidium sp., Aureobasidium sp., Mucor sp., Penicillum sp., Ulocladium sp. and Rhizopus sp.

Concerning surfaces, 21 different species of fungi were identified, being the 4 genera more identified Penicillium sp. (51,8\%), Cladosporium sp. (25,4\%), Alternaria sp. (6,1\%) and Aspergillus sp. (4,2\%). Among Aspergillus genus, were identified the species Aspergillus glaucus, Aspergillus. fumigatus and Aspergillus niger. In addition to these genera, were also identified: Cladosporium sphaerosperma, Chrysosporium sp., Trichothecium roseum, Graphium sp., Scopulariopsis sp., Fusarium oxysporum, Trichoderma sp., Exophiala sp., Chrysonilia sp., Scytalidium sp., Gliocladium sp., Ulocladium sp., Mucor sp. and Scedosporium prolificans sp. (Table 1).

Regarding comparison of concentrations found in air, for indoor and exterior environments, all indoor areas showed less contamination than exterior areas. However, all the indoor spaces presented fungal species different from the ones isolated outdoor. Some fungi that were only isolated indoor were: Aspergillus flavus, Aspergillus niger, Aspergillus fumigatus, Phoma sp., Aureobasidium sp., Mucor sp., Fusarium clamidosporos sp., Fusarium incarnatum, Fusarium oxysporum and Rhizopus sp..

Outside premises Cladosporium, Alternaria and Chrysosporium were the prevailing genera.

Concerning quantitative results the highest fungal contamination found indoor was $240 \mathrm{CFU} / \mathrm{m}^{3}$, and outside premises was $740 \mathrm{CFU} / \mathrm{m}^{3}$. 
48 Environmental Toxicology III

Table 1: $\quad$ Most frequent fungi identified in the poultry air and surfaces.

\begin{tabular}{|c|c|}
\hline Air & Frequency (\%) \\
\hline Cladosporium sp. & 40,5 \\
Alternaria sp. & 10,8 \\
Chrysosporium sp. & 6,8 \\
Aspergillus sp. & 6,8 \\
Mucor sp. & 2,7 \\
Penicillium sp. & 2,7 \\
Scytalidium sp. & 2,7 \\
Others & 27 \\
\hline Surfaces & Frequency (\%) \\
\hline Penicillium sp. & 51,8 \\
Cladosporium sp. & 25,4 \\
Alternaria sp. & 6,1 \\
Aspergillus sp. & 4,2 \\
Chrysosporium sp. & 2,9 \\
Others & 9,6 \\
\hline
\end{tabular}

Regarding the influence of environmental variables monitored no significant correlation $(p>0,05)$ was reveal. Temperature and relative humidity contributed only in $2,75 \%$ and $59,0 \%$, respectively, to $\mathrm{CFU} / \mathrm{m}^{3}$ variation explanation (Figure 1 and Figure 2).

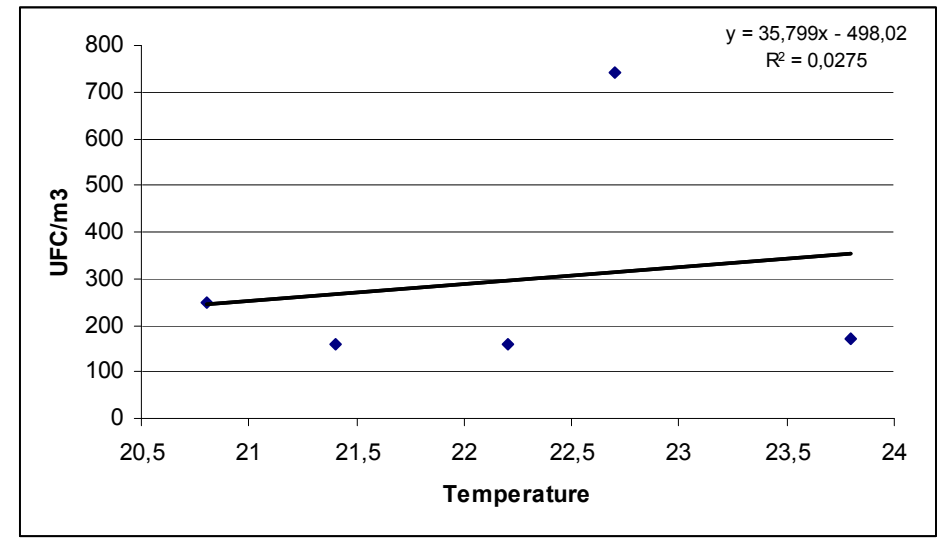

Figure 1: Influence of temperature in $\mathrm{CFU} / \mathrm{m}^{2}$. 


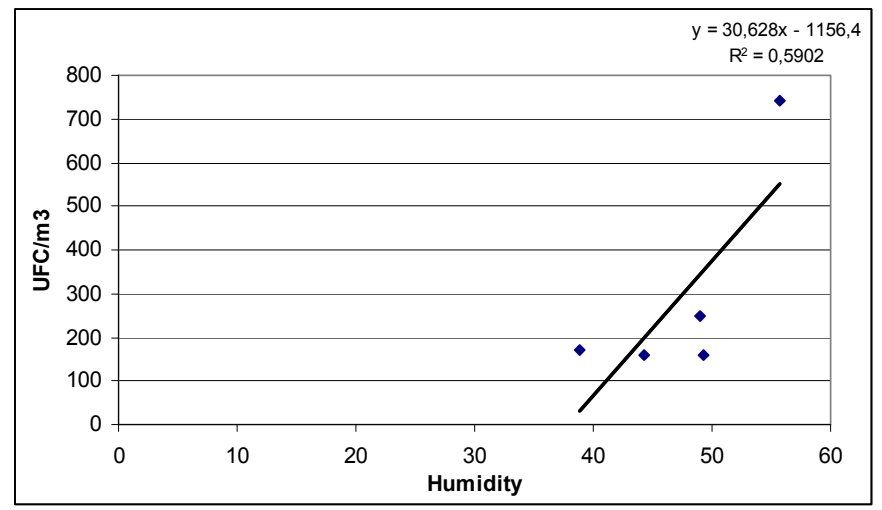

Figure 2: $\quad$ Influence of relative humidity in $\mathrm{CFU} / \mathrm{m}^{2}$.

\section{Discussion}

Cladosporium genus, predominant type in the air, is probably the fungus that occurs more frequently around world, especially in temperate climates [12]. The same genus is deeply connected to indoor condensation problems [13]. For Penicillium sp., predominant type in the surfaces, there are different potential risks associated with their inhalation, due to the toxins release. In Alternaria sp. case, second in air and third in surfaces, there are potentially allergic effects, only because the spores presence [14].

With regard to qualitative assessment of fungal contamination in air, it is suggested that, among other species, Aspergillus fumigatus and Penicillium, Trichoderma, Fusarium and Ulocladium species, all of them isolated in the present study, are regarded as indicators of humidity problems or potential risk to health [1]. Moreover, according to American Industrial Hygiene Association (AIHA), in 1996, for determination of biological contamination in environmental samples, confirmed presence of the species Aspergillus flavus and Aspergillus fumigatus, both identified in this study, requires implementation of corrective measures [15]. Also noteworthy is the fact that Aspergillus fumigatus, isolated in air and surfaces, is one of the saprophytic fungi most widespread in air and is capable of causing severe or sometimes fatal aspergillosis [16].

In addition, Aspergillus flavus, also isolated in the poultry air, is a wellknown producer of potent mycotoxins (aflatoxins) and besides this species other isolated genera, like Fusarium and Penicillium, are also known as mycotoxins producers [15].

For quantitative assessment in air $\left(\mathrm{CFU} / \mathrm{m}^{3}\right)$ is proposed corrective measures implementation whenever, in a given space, one or more of the following conditions were verified: a) $>50 \mathrm{CFU} / \mathrm{m}^{3}$ of a single fungal species; b) $>150$ $\mathrm{CFU} / \mathrm{m}^{3}$ if several fungal species are isolated; c) $>300 \mathrm{CFU} / \mathrm{m}^{3}$ if there are mainly filamentous fungi [17]. The first condition a) was found for Cladosporium sp. and Exophiala sp. and the second condition b) was found in all 
the interior spaces monitored. However, and because fungal concentration indoor was considerably lower than one outdoor, some authors consider that there should be no concerns about a possible fungal contamination [14]. Taking into account what is mentioned in Portuguese law, $500 \mathrm{CFU} / \mathrm{m}^{3}$ maximum reference concentration in interior air, was not exceeded in any of the monitored spaces. In one interior space, where $240 \mathrm{CFU} / \mathrm{m}^{3}$ were isolated, revealed the highest fungal contamination.

Also worth mentioning is the fact that outdoor air is a major source of fungi in indoor, thus justifying the coincidence between prevailing genera, Cladosporium, Alternaria and Chrysosporium, in both these environments [18]. Nonetheless, all monitored interior spaces had fungal species different from the ones isolated outside, suggesting fungal contamination from within [19].

Surfaces sampling, in addition to air sampling, is essential to achieve the fungal contamination characterization and evaluation, and can be used to identify contamination sources $[20,21]$. There was some coincidence between species isolated in air and on surfaces and, furthermore, should be taken into account that species present only on surfaces may aerosolized and become airborne, depending on activities carried out [22] and its occupants [23], whereas in this case will be not only farmers, but also chickens.

Taking into account the isolated fungal species in poultry air and surfaces, we have to consider not only the occupational health problem, due to the Aspergillus fumigatus presence, among others species, but also the public health problem since there were some fungal species that release mycotoxins [15], and there is evidence that mycotoxins can cause human disease from inhalation and from ingestion of fungus-contaminated food [9]. Besides that, early studies did provide data illustrating that increasing human hepatocellular carcinoma rates corresponded to increasing levels of dietary aflatoxins exposure [24]. The requirements for aflatoxin production are relatively non-specific, since moulds can produce them on almost any foodstuff, with therefore a wide range of commodities contaminated at final concentrations which can vary from $<1 \mu \mathrm{g} / \mathrm{kg}$ (1 p.p.b.) to $12000 \mu \mathrm{g} / \mathrm{kg}$ (12 p.p.m) [25]. Because measurement of human exposure to aflatoxin, by sampling foodstuffs or by dietary questionnaires is extremely imprecise, it's important to consider aflatoxin exposure biomarkers since they have great potential for accurate assessment of exposure [26].

Results related to environmental variables are not consistent with the expected [27]. It was found that the relationship between the fungal air contamination and the temperature and relative humidity was not statistically significant $(p>0,05)$. This may be justified by the effect of other environmental variables also influencing fungal spreading, namely workers and chickens, who may carry, in their own body (commensal flora) or clothing, a great diversity of fungal species [23], as well the developed activities that may also affect fungal concentration [22]. 


\section{Conclusions}

It was possible to characterize fungal distribution in poultry air and surfaces, evaluate the association of environmental variables with this distribution and recognize the public heath problem, because there is scientific evidence that mycotoxins can cause human disease after inhalation or ingestion of funguscontaminated food. Furthermore, also worth mentioning is the occupational health problem due to the presence of Aspergillus fumigatus in poultry air and surfaces.

Unlike other studies, environmental variables monitored (temperature and relative humidity) did not show the expected association with fungal concentration, which may possibly have resulted from other variables not investigated in this study.

\section{References}

[1] Goyer N, Lavoie J, Lazure L \& Marchand G., Bioaerosols in the Workplace: Evaluation, Control and Prevention Guide. Institut de Recherche en Santé et en Sécurité du Travail du Québec, 2001.

[2] Daisey J, Angell W \& APTE M., Indoor air quality, ventilation and health symptoms in schools: an analysis of existing information. Indoor Air, 13, pp., $53-64,2003$.

[3] Jarvis B., Stachybotrys chartarum: a fungus of our time. Phytochemistry, 64, pp. $53-60,2003$.

[4] Molocznik A., Qualitative and quantitative analysis of agricultural dust in working environment. Ann. Agric. Environm. Med., 9, pp., 71 - 78, 2002.

[5] Buys E, Nortjé G, Jooste P \& Von Holy A., Bacterial population associated with bulk-packaged beef supplemented with dietary vitamin E. Int. J. Food. Microbiol; 50, pp., 239 - 244, 2000.

[6] Borch E \& Arinder P., Bacteriological safety issues in red meat and readyto-eat meat products, as well as control measures. Meat Sci., 62, pp., $381-$ 390, 2002.

[7] White P, Collins J, McGill K, Monahan C \& O’Mahony H., Distribution and prevalence of airborne microorganisms in three commercial poultry processing plants. J. Food Products, 64, pp., 388 - 391, 2001.

[8] Lues J, Theron M, Venter P \& Rasephei M., Microbial composition in bioaerosols of a high-throughput chicken-slaughtering facility. Poultry Science, 86, pp., 142 - 149, 2007.

[9] Shlosberg A, Zadikov I, Perl S, Yakobson B, Varod Y \& Elad D., Aspergillus clavatus as the probable cause of a lethal mass neurotoxicosis in sheep. Mycopathology, 114 (1), pp., 35 - 9, 1991.

[10] Rao C, Burge H \& Chang J., Review of quantitative standards and guidelines for fungi in indoor air. J Air Waste Manage Assoc., 46, pp. 899 908, 1996.

[11] Hoog C, Guarro J, Gené G \& Figueiras M., (2 $2^{\text {th }}$ ed). Atlas of Clinical Fungi. Centraalbureau voor Schimmelcultures, 2000. 
[12] Cooley J, Wong W, Jumper C \& Straus D., Correlation between the prevalence of certain fungi and sick building syndrome. Occup. Environ Med, 55, pp., $579-584,1998$.

[13] Garret M, Rayment P, Hooper M, Abranson M \& Hooper B., Indoor Airborne Fungal Spores, House Dampness and Associations with Environmental Factors and Respiratory Health in Children. Clinical and Experimental Allergy, 28, pp., 459 - 467, 1998.

[14] Kemp P, Neumeister-Kemp H, Esposito B, Lysek G \& Murray F., Changes in airborne fungi from the outdoors to indoor air; Large HVAC systems in nonproblem buildings in two different climates. American Industrial Hygiene Association, 64, pp. 269 - 275, 2003.

[15] American Industrial Hygiene Association: Field Guide for the Determination of Biological Contaminants in Environmental Samples. AIHA, 1996.

[16] Yao M \& Mainelis G., Analysis of Portable Impactor Performance for Enumeration of Viable Bioaerosols. Journal of Occupational and Environmental Hygiene, 4, pp., 514 - 524, 2007.

[17] Miller J., Fungi as Contaminants of Indoor Air. Atmos. Environm, 26 A, pp., 2163-2172, 1992.

[18] Nevalainen A., Bio-aerosols as exposure agents in indoor environment in relation to asthma and allergy. Section 3 Asthma and allergy. Proceedings of the First ENVIE Conference on Indoor Air Quality and Health for EU Policy, Helsinki, Finland, 2007.

[19] Kemp P, Neumeister-Kemp H, Murray F \& Lysek G., Airborne fungi in non-problem buildings in a southern-hemisphere Mediterranean climate: preliminary study of natural and mechanical ventilation. Indoor and Built Environment, 11; pp., 44 - 53, 2002.

[20] Stetzenbach L, Buttner M \& Cruz P., Detection and enumeration of airborne biocontaminants. Current Opinion in Biotechnology, 15, pp. $170-$ 174, 2004.

[21] Klánová K \& Hollerová J., Hospital indoor environment: screening for micro-organisms and particulate matter. Indoor and Built Environment, 12, pp. $61-67,2003$.

[22] Buttner M \& Stetzenbach L., Monitoring Airborne fungal spores in an experimental indoor environment to evaluate sampling methods and the effects of human activity on air sampling. Applied and Environmental Microbiology, 59, pp. 219-226, 1993.

[23] Scheff P, Pulius V, Curtis L \& Conroy L., Indoor air quality in a middle school, Part II: Development of emission factors for particulate matter and bioaerosols. Applied Occupational and Environmental Hygiene, 15, pp. 835 $-842,2000$.

[24] Bosch F \& Munoz N., Prospects for epidemiological studies on hepatocellular cancer as a model for assessing viral and chemical interactions. IARC Scientific Publications, 89, pp. 427 - 438, 1988. 
[25] Ellis W, Smith J, Simpson B \& et al., Aflatoxins in food - occurrence, biosynthesis, effects on organisms, detection, and methods of control. Crit Rev Food Sci Nutr, 30, pp. 403 - 439, 1991.

[26] Wild C \& Turner P., The toxicology of aflatoxins as a basis for public health decisions. Mutagenesis, 17, pp. $471-481,2002$.

[27] Kakde U, Kakde H \& Saoji A., Seasonal Variation of Fungal Propagules in a Fruit Market Environment, Nagpur (India). Aerobiologia, 17, pp. 177 $182,2001$. 